\title{
INTERVENÇÃO NUTRICIONAL NAS DOENÇAS CRÔNICAS NÃO TRANSMISSÍVEIS: UMA REVISÃO DE LITERATURA
}

\section{NUTRITIONAL INTERVENTION IN NON-COMMUNICABLE CHRONIC DISEASES: A LITERATURE REVIEW}

Ana Cristina Santos Costa1 ${ }^{1}$ Milene Oliveira Alves², Patrícia Danielle Antunes ${ }^{3}$, Kássia Héllen Vieira ${ }^{4}$

\section{RESUMO}

A intervenção nutricional é de grande relevância para a redução dos riscos do desenvolvimento das Doenças Crônicas Não Transmissíveis (DCNT), é um importante meio de controle e prevenção de suas complicações secundárias à saúde. Diante do exposto, o objetivo do estudo foi analisar a importância da intervenção nutricional na prevenção das DCNT através da leitura de artigos científicos. Este estudo trata-se de uma revisão sistemática de literatura, realizada através da leitura de artigos publicados em revistas e jornais nas bases de dados Pubmed, Scielo, Lilacs, Bireme e sites de informações, considerando a relevância informativa. Após a seleção dos artigos foi realizada a coleta de dados através da leitura exploratória. A adoção de uma dieta mediterrânea enriquecida com azeite de oliva extra-virgem apresentou maior redução dos riscos de diabetes entre pessoas com alto risco cardiovascular, devido ao alto teor de compostos anti-inflamatórios e antioxidantes. A prática de atividade física e uma alimentação equilibrada, pobre em gorduras saturadas demonstraram efeitos na redução de medidas antropométricas, níveis glicêmicos e pressóricos. Os estudos evidenciaram a influência e a importância de intervenções nutricionais voltadas para a prevenção, controle e redução das complicações das DCNT.

Palavras-chave: Doenças não transmissíveis. Fatores de risco. Comportamento alimentar. Prevenção de doenças.

\section{ABSTRACT}

Nutritional intervention is of great relevance for reducing the risks of the development of Chronic Noncommunicable Diseases (NCD), it is an important means of controlling and preventing its secondary health complications. Given the above, the objective of the study was to analyze the importance of nutritional intervention in the prevention of NCD through the reading of scientific articles. This study is a systematic review of the literature, carried out by reading articles published in magazines and newspapers in the databases Pubmed, Scielo, Lilacs, Bireme and information sites, considering the informational relevance. After selecting the articles, data were collected through exploratory reading. The adoption of a Mediterranean diet enriched with extra virgin olive oil showed a greater reduction in the risk of diabetes among people at high cardiovascular risk, due to the high content of anti-inflammatory and antioxidant compounds. The practice of physical activity and a balanced diet, low in saturated fats demonstrated effects in the reduction of anthropometric measurements, blood glucose and blood pressure levels. Studies have shown the influence and importance of nutritional interventions aimed at the prevention, control and reduction of NCD complications.

Keywords: Non-communicable diseases. Risk factors. Eating behavior. Disease prevention.
${ }^{1}$ Nutricionista pela FASI e Pósgraduada em Nutrição e Metabolismo na Prática Clínica e Desportiva pela FUNORTE.

E-mail:

cristi_artes@yahoo.com.br

${ }^{2}$ Nutricionista pela FASI e Pósgraduada em Nutrição e Metabolismo na Prática Clínica e Desportiva pela FUNORTE.

${ }^{3}$ Nutricionista pela FASI ne Pósgraduada em Nutrição e Metabolismo na Prática Clínica e Desportiva pela FUNORTE.

Nutricionista e Mestre em Ciência e Tecnologia de Alimentos. Docente do curso de Bacharel em Nutrição da FASI e FUNORTE. 


\section{INTRODUÇÄO}

Das últimas décadas até os momentos atuais ocorreram mudanças sociais e econômicas que refletiram no perfil nutricional da população, onde prevalecia as doenças infecciosas e desnutrição, o cenário foi gradualmente substituído pelas Doenças Crônicas não Transmissíveis (DCNT). ${ }^{1}$

Indícios apontam que o crescimento das DCNT está relacionado ao aumento dos principais fatores de risco como: consumo desenfreado de alimentos não saudáveis associados ao sedentarismo, uso prejudicial do álcool tabagismo e obesidade ${ }^{2}$. Pode estar também relacionada ao envelhecimento da sociedade e a fatores sociais como: má qualidade de vida, desigualdade social, baixa escolaridade, pouco acesso à informação e ser pertencente à grupo susceptível. ${ }^{3}$

As doenças não transmissíveis relacionadas à obesidade levam a milhões de mortes em todo o mundo. A obesidade é caracterizada como epidemia desafiadora, é considerada importante fator de risco por estar associada às principais DCNT. ${ }^{4}$

Para Gonçalves et al. ${ }^{5}$ o índice de obesidade cresce de maneira alarmante em vários países, inúmeros fatores são apontados como principais causas para o seu desenvolvimento, como exemplo as alterações do próprio envelhecimento, mudanças no estilo de vida, a falta de atividade física, consumo de alimentos com elevada densidade energética, influência dos fatores sociais, econômicos e ambientais.

O sobrepeso e obesidade desde a infância pode acarretar aumento dos casos de doenças crônicas não transmissíveis. Estratégia de prevenção precoce como exemplo, a nutrição adequada durante a gestação, infância e adolescência podem-se obter resultados relevantes na prevenção das DCNT na fase adulta. ${ }^{6}$

Conforme Silva et al..$^{7}$ os fatores de risco para o desenvolvimento da hipertensão arterial podem estar relacionados com a predisposição genética, a idade, sexo, o consumo excessivo de bebidas alcoólicas, tabagismo, sobrepeso ou a obesidade devido ao estilo de vida pouco saudável e o estresse. A hipertensão arterial está na maioria das vezes referente a distúrbios metabólicos, é a doença circulatória que mais prevalece, está relacionada principalmente à alimentação inadequada com ingestão elevada de sal. ${ }^{8}$

O Diabetes Mellitus (DM) é uma doença crônico-degenerativa, de origem multifatorial, sendo identificado pelo aumento dos níveis glicêmicos, resultante da ausência ou da incapacidade da insulina desempenhar suas funções, acarretando várias 
complicações e enfermidades aos órgãos essenciais. As principais causas de risco para o diabetes estão relacionadas com o excesso de peso, pressão alta, hereditariedade, sedentarismo, dieta pouco saudável com consumo de alimentos industrializados e gordurosos, e ainda a intolerância à glicose. ${ }^{9}$

Atualmente, o diabetes está entre as principais causas que levam as morbimortalidades, apresentando grande problema de saúde pública. Estima-se que 382 milhões de pessoas têm DM (8,3\%), esse número poderá aumentar até 592 milhões em $2035 .{ }^{10}$

No estudo de Mitcha et al. ${ }^{11}$ estimou-se que os fatores alimentares estão relacionados a uma parcela considerável de mortalidade por doenças cardiovasculares, derrames e diabetes tipo 2.

A prática dietética cuidadosa é essencial no tratamento secundário de doenças como diabetes e hipertensão, e importante meio de controle dessas DCNT e de suas complicações secundárias à saúde, uma vez que essas doenças geralmente resultam de estilos de vida não adequado. ${ }^{12} 13$ As orientações nutricionais e mudança no estilo de vida são essenciais para conseguir esse controle e ainda reduzir prováveis riscos de complicações relacionadas a essas patologias. ${ }^{3}$

Conforme Garg et al. ${ }^{14}$ as intervenções como redução do peso corporal, pressão arterial, colesterol, glicemia, dieta balanceada, bem-estar mental, abstinência do fumo e exercícios regulares têm demonstrado um impacto benéfico sobre os principais fatores de risco das DCNT.

Neste contexto, o principal objetivo desse estudo foi analisar, por meio de uma revisão sistemática da literatura, a importância e os impactos da intervenção nutricional na prevenção das doenças crônicas não transmissíveis.

\section{MATERIAIS E METODOS}

Este estudo trata-se de uma revisão sistemática de literatura, que foi realizada através da leitura de artigos publicados em revistas e jornais nas bases de dados Pubmed, Scielo, Lilacs, Bireme e sites de informações, considerando a relevância informativa. $O$ idioma não foi limitado para ter mais abrangência do referencial teórico.

A amostra foi constituída por artigos científicos sobre o tema proposto e/ou que contenham os seguintes descritores: doenças não transmissíveis, fatores de risco, comportamento alimentar, prevenção de doenças, para maior credibilidade da busca. 
DOI: 10.18606/2318-1419/amazonia.sci.health.v9n1p96-104 Revista Amazônia Science \& Health
COSTA, ACS; ALVES, MO; ANTUNES, PD; VIEIRA, KH.

Intervenção Nutricional nas Doenças Crônicas Não Transmissíveis: Uma Revisão de Literatura.

A leitura e seleção dos artigos foi feita no ano de 2019, priorizando os materiais técnicos científicos publicados em até cinco anos antes do ano de início da coleta (2014 a 2019).

Foram utilizados para coleta de material, artigos publicados em jornais, revistas científicas, e sites de informações e considerados os artigos que apresentaram assuntos referentes ao tema proposto e que corresponderam ao período selecionado de 2014 a 2019, e desconsiderados os que não apresentaram assuntos relacionados e não contemplaram ao período estipulado.

\section{RESULTADOS E DISCUSSÃO}

Após a leitura, os artigos foram selecionados de acordo com os critérios de inclusão e exclusão. Estudos internacionais corresponderam a $60 \%$ da pesquisa. Os nacionais compreenderam em $40 \%$. Dos 20 artigos selecionados, seis foram estudos que abordaram intervenção nutricional, onde somente um destes artigos escolhidos é de estudo nacional. Os dados descritivos mais relevantes dos seis estudos selecionados encontram -se na Tabela 1, foram descritos os dados como: o nome do autor e ano de publicação; local; ano da realização do estudo; número de amostra; metodologia e intervenção aplicada.

Esperava-se encontrar mais artigos relacionado a intervenções na hipertensão, os que foram encontrados abordaram mais sobre as intervenções na diabetes e obesidade, intervenções de educação nutricional em ambiente escolar e estudos de revisão de literatura e meta-análise.

Tabela 1- Dados descritivos dos estudos selecionados.

\begin{tabular}{|c|c|c|c|c|c|}
\hline $\begin{array}{l}\text { Autor e ano } \\
\text { da publicação }\end{array}$ & $\begin{array}{l}\text { Local da } \\
\text { realizaçã } \\
\text { o do } \\
\text { estudo }\end{array}$ & $\begin{array}{l}\text { Ano de } \\
\text { realização } \\
\text { do estudo }\end{array}$ & Amostra & $\begin{array}{c}\text { Metodologia } \\
\text { utilizada }\end{array}$ & $\begin{array}{c}\text { Intervenção nutricional } \\
\text { aplicada }\end{array}$ \\
\hline $\begin{array}{l}\text { Salas-Salvadó } \\
\text { et al. }{ }^{15}\end{array}$ & Espanha & $\begin{array}{c}2003 a \\
2010\end{array}$ & 3541 & $\begin{array}{l}\text { Estudo } \\
\text { multicêntrico, } \\
\text { randomizado. }\end{array}$ & $\begin{array}{c}\text { Dieta Mediterrânea } \\
\text { suplementada com azeite } \\
\text { de oliva extra virgem } \\
\text { (EVOO) }\end{array}$ \\
\hline $\begin{array}{l}\text { Diabetes } \\
\text { Prevention } \\
\text { Program } \\
\text { Research } \\
\text { Group }^{16}\end{array}$ & EUA & 2014 & 2776 & $\begin{array}{l}\text { Estudo clínico } \\
\text { controlado e } \\
\text { randomizado }\end{array}$ & $\begin{array}{c}\text { Dieta saudável com baixo } \\
\text { teor de gordura e baixas } \\
\text { calorias }\end{array}$ \\
\hline Radovanovic $^{17}$ & $\begin{array}{c}\text { Paraná- } \\
\text { Brasil }\end{array}$ & 2012 & 94 & $\begin{array}{l}\text { Ensaio clínico } \\
\text { randomizado }\end{array}$ & $\begin{array}{c}\text { Intervenção } \\
\text { multiprofissional }\end{array}$ \\
\hline
\end{tabular}


DOI: 10.18606/2318-1419/amazonia.sci.health.v9n1p96-104 Revista Amazônia Science \& Health
COSTA, ACS; ALVES, MO; ANTUNES, PD; VIEIRA, KH.

Intervenção Nutricional nas Doenças Crônicas Não Transmissíveis: Uma Revisão de Literatura.

\begin{tabular}{|c|c|c|c|c|c|}
\hline $\begin{array}{c}\text { Camolas et } \\
\text { al. }^{18}\end{array}$ & $\begin{array}{l}\text { Lisboa- } \\
\text { Portugal }\end{array}$ & 2015 & $\begin{array}{l}25 \text { por grupo de } \\
\text { indivíduos com } \\
\text { obesidade } 3 \text { ou } \\
2 \text { com comorbi- } \\
\text {-dades. }\end{array}$ & $\begin{array}{l}\text { Ensaio clínico } \\
\text { aleatorizado e } \\
\text { cego }\end{array}$ & $\begin{array}{l}\text { Intervenção nutricional } \\
\text { protocolada, direcionada a } \\
\text { mudança de estilo de vida } \\
\text { através do método } \\
\text { INDIVÍDUO. }\end{array}$ \\
\hline Honsek et al. ${ }^{19}$ & Berlim & $\begin{array}{c}03 / 2010 a \\
10 / 2012\end{array}$ & 180 & $\begin{array}{l}\text { Estudo de } \\
\text { intervenção } \\
\text { prospectivo, } \\
\text { randomizado, } \\
\text { duplo cego }\end{array}$ & $\begin{array}{l}\text { Suplementação de extrato } \\
\text { de fibra insolúvel de aveia } \\
\text { e intervenção alimentar. }\end{array}$ \\
\hline Li et al. ${ }^{20}$ & $\begin{array}{l}\text { Estado } \\
\text { Unidos }\end{array}$ & $\begin{array}{c}1980 a \\
2010\end{array}$ & $\begin{array}{c}84.628 \\
\text { mulheres e } \\
\text { 42.908 homens }\end{array}$ & $\begin{array}{l}\text { Estudo } \\
\text { prospectivo de } \\
\text { coorte }\end{array}$ & $\begin{array}{c}\text { Questionário } \\
\text { semiquantitativo de } \\
\text { frequência alimentar a cada } \\
4 \text { anos. }\end{array}$ \\
\hline
\end{tabular}

Fonte: Autoria Própria (2019).

Salvadó Salas et al. ${ }^{15}$ concluiu nos seus estudos onde indivíduos foram designados aleatoriamente e estratificados por local, sexo e idade, mas não status de diabetes, para receber 1 de 3 dietas: dieta mediterrânea suplementada com azeite de oliva extra virgem (EVOO), dieta mediterrânea suplementada com nozes ou uma dieta controle (conselhos sobre uma dieta com pouca gordura) em comparação com estas duas últimas a dieta mediterrânea enriquecida com azeite de oliva extra virgem (EVOO) teve maior redução dos riscos de diabetes entre pessoas com alto risco cardiovascular.

A dieta mediterrânea tradicional é caracterizada pelo alto consumo de grãos e cereais, oleaginosas, hortaliças, frutas e azeite de oliva, moderada ingestão de peixe e álcool, especialmente o vinho; e baixo consumo de lácteos, carnes e doces. ${ }^{21}$ Devido ao alto teor de gorduras saturas, compostos e vitaminas antioxidantes (como as vitaminas $A, C$ e $E$ ), componentes bioativos e anti-inflamatórios (como o ômega 3) presentes nesta dieta reduzem o risco de doenças cardiovasculares, exercendo efeitos benéficos sobre a adiposidade corporal, resistência à insulina e outros distúrbios metabólicos. ${ }^{22}$

O DPPOS (Diabetes Prevention Program Outcomes Study) ${ }^{16}$ mostrou que os participantes do Programa de Mudança de Estilo de Vida do DPP (Diabetes Prevention Program) ou a metformina, reduziram ou atrasaram o diabetes tipo 2 por pelo menos 15 anos. Neste estudo comparou a metformina em $850 \mathrm{mg}$ duas vezes por dia ou um programa individual de intervenção no estilo de vida comportamental com placebo. O programa de estilo de vida incluiu um currículo de 16 sessões com sessões individuais destinadas a alcançar uma perda de peso de $7 \%$ através de uma dieta saudável com baixo teor de gordura e baixas calorias e 150 minutos por semana de atividade física de intensidade moderada. Concluiu-se que os benefícios da intervenção no estilo de vida, além da 
prevenção ou atraso do diabetes observado até o momento, incluem uma redução nos fatores de risco para doenças cardiovasculares e síndrome metabólica, prevalência reduzida de sintomas do trato urinário inferior associados à obesidade e diabetes e melhor qualidade de vida.

Para Radovanovic et al. ${ }^{17}$ o resultado da intervenção mostrou-se efetivo, as orientações em saúde e nutricionais juntamente aos exercícios físicos com uma equipe multiprofissional, mostraram-se adequados e eficazes na redução e/ou controle dos valores da pressão, bioquímicos e antropométricos, principalmente para aqueles que participaram de todo o processo da intervenção. Esta proposta de intervenção é de extrema importância e viável para o trabalho da equipe multiprofissional junto a pessoas com DCNT.

Uma das intervenções nutricionais que exerce efeito de redução e/ou controle dos níveis pressóricos é o padrão dietético DASH (Dietary Approaches to Stop Hypertension), que apresenta um alto teor de frutas e hortaliças, um teor moderado de lácteos e menor teor de gordura e proteína animal, porém com uma quantidade considerável de proteínas vegetais. ${ }^{23}$

Nos estudos de Camolas et al. ${ }^{18}$, apesar da duração de somente 6 meses, pode apontar expectativas na efetividade da intervenção nutricional pelo método INDIVIDUO no acompanhamento dos pacientes com obesidade, onde os pacientes participantes foram direcionados a mudanças no estilo de vida através de estratégias fundamentadas que tiveram como resultado a melhoria clínica e da qualidade de vida dos mesmos.

Já neste estudo de Honsek et al. ${ }^{19}$ foi comparado o efeito de um extrato insolúvel de fibra de aveia com o placebo no metabolismo da glicose e na incidência de diabetes, ele concluiu que atualmente, não se pode fornecer fortes evidências de um efeito benéfico do metabolismo onglicêmico da fibra insolúvel em cereais, embora estudos adicionais possam apoiar efeitos menores da suplementação de fibras na redução dos níveis de glicose, resistência à insulina e incidência de diabetes tipo 2 .

Li et al. ${ }^{20}$ procurou investigar através do estudo prospectivo de coorte as associações de gorduras saturadas em comparação com gorduras insaturadas e diferentes fontes de carboidratos em relação ao risco de doença cardíaca coronária (DCC), acompanhando um grupo de homens e mulheres durante 24 e 30 anos, avaliando a dieta pelo questionário semiquantitativo de frequência alimentar a cada 4 anos. Os resultados indicaram que gorduras insaturadas, especialmente os ácidos graxos poli-insaturados e/ou carboidratos de alta qualidade de grãos integrais, podem ser usados para substituir gorduras saturadas 
DOI: 10.18606/2318-1419/amazonia.sci.health.v9n1p96-104 Revista Amazônia Science \& Health
COSTA, ACS; ALVES, MO; ANTUNES, PD; VIEIRA, KH.

Intervenção Nutricional nas Doenças Crônicas Não Transmissíveis: Uma Revisão de Literatura.

e reduzir o risco de DCC. Por outro lado, carboidratos de amidos refinados, açúcares adicionados foram associados positivamente a um risco de doença coronariana.

\section{CONSIDERAÇÕES FINAIS}

De modo geral a maior parte dos estudos selecionados abordaram sobre as DCNT e mostraram a influência e a importância da intervenção nutricional voltadas para a prevenção, controle e redução das complicações das doenças crônicas não transmissíveis.

A adoção da dieta mediterrânea, por exemplo, enriquecida com azeite de oliva extravirgem favoreceu à redução do risco de desenvolvimento de diabetes. Em suma, intervenções nutricionais que visão a manutenção do peso saudável e de um estilo de vida ativo e alimentação com baixo teor de gordura saturada, alto teor de gorduras poliinsaturadas, rica em fibras, frutas e vegetais reduziu parâmetros antropométricos, níveis pressóricos e parâmetros bioquímicos relacionados ao aumento do risco cardiovascular e de desenvolvimento de doenças como o diabetes, hipertensão arterial, obesidade e síndrome metabólica

Durante a pesquisa exploratória pode-se perceber a dificuldade em encontrar estudos nacionais recentes, pois como critério de inclusão os artigos deveriam compreender 0 período de 2014 a 2019, sendo um fator limitante deste estudo de revisão.

Espera-se que mais estudos possam ser realizados com intuito de evidenciar a relevância da intervenção nutricional na prevenção e tratamento das DCNT, avaliando a história pregressa e familiar dos participantes, monitoração de exames bioquímicos como hemoglobina glicosilada, insulina basal, cortisol e mapeamento genético voltado para averiguação de genes que podem estar envolvidos com a predisposição de desenvolver alguma dessas doenças e que podem ser superestimulados devido ao estilo de vida e alimentação inadequada.

\section{REFERÉNCIAS}

1. Claro RM, Santos MAS, Oliveira TP, Pereira CA, Szwarcwald CL, Malta, D. C. Consumo de alimentos não saudáveis relacionados a doenças crônicas não transmissíveis no Brasil: Pesquisa Nacional de Saúde, 2013. Epidemiol Serv Saúde. 2015; 24(2):257-265.

2. Malta DC, Bernal RTI, Lima MG, Araújo SSC, Silva MMA, Freitas MIF, et al. Doenças crônicas não transmissíveis e a utilização de serviços de saúde: análise da Pesquisa Nacional de Saúde no Brasil. Rev Saúde Públ. 2017;51(1):1-10. 
3. Oliveira SKM, Caldeira AP. Fatores de risco para doenças crônicas não transmissíveis em quilombolas do norte de Minas Gerais. Cad Saude Colet. 2016;24(4):420-427.

4. Banjare JB, Bhalerao S. Obesity associated noncommunicable disease burden. Int J Health Allied Sci. 2016;5(2):81-87.

5. Gonçalves JTT, Silveira MF, Campos MCC, Costa LHR. Sobrepeso e obesidade e fatores associados ao climatério. Cien Saude Colet. 2016;21(4):1145 - 1155.

6. Duarte AS, Christofoli MC, Pontin B, Paludo J. Síndrome metabólica na infância e adolescência: uma revisão. Rev Bras Nutr Clín. 2015;30(2):170- 173.

7. Silva EC. Prevalência de hipertensão arterial sistêmica e fatores associados em homens e mulheres residentes em municípios da Amazônia Legal. Rev Bras Epidemiol. 2016;19(1):38-51.

8. Malta DC, Bernal RTI, Andrade SSCA, Silva MMA, Velasquez-Melendez G. Prevalência e fatores associados com hipertensão arterial autorreferida em adultos brasileiros. Rev Saúde Públ. 2017;51(1):1-11.

9. Lima CLJ, Ferreira TMC, Oliveira PS, Ferreira JDL, Silva EC, Costa MML. Caracterização de usuários em risco de desenvolver diabetes: um estudo transversal. Rev Bras Enferm. 2018;71(1):516-523.

10. Guariguata L, Badejo DR, Hambleton I, Beagley J, Linnenkamp U, Shaw JE. Global estimates of diabetes prevalence for 2013 and projections for 2035 Diabetes Res Clin Pract. 2014;103(2):137-149.

11. Mitcha R, Peñalvo JL, Cudhea F, Imamura F, Rehm CD, Mozaffarian D. Association between dietary factors and mortality from heart disease, stroke, and type 2 diabetes in the United States. JAMA. 2017;317(9):912-924.

12. Ozcariz SG, Bernardo CO, Cembranel F, Peres MA, González-Chica DA. Dietary practices among individuals with diabetes and hypertension are similar to those of healthy people: a population-based study. BMC Public Health. 2015;15(479):2-10.

13. Cembranel F, Bernardo CO, Ozcariz SGIQ, D'ors E. Impact of the diagnosis of diabetes and/or hypertension on healthy food consumption indicators: a longitudinal study of elderly persons. Rev Bras Geriatr Gerontol.2017;20(1):34-46.

14. Garg A, Anand T, Sharma U, Kishore J, Chakraborty M, Ray PC, Ingle, G.K. Prevalence of risk factors for chronic non-communicable diseases using WHO steps approach in an adult population in delhi. J Family Med Prim Care. 2014;3(2):112-118.

15. Salas-Salvadó J, Bulló M, Estruch R, Ros E, Covas Ml, Ibarrola-Jurado N, et al. Prevention of diabetes with Mediterranean diets: a subgroup analysis of a randomized trial. Ann Intern Med. 2014;160(1):1-102, 2014. 
16. Diabetes Prevention Program Research Group. Long-term effects of lifestyle intervention or metformin on diabetes development and microvascular complications over 15-year follow-up: the Diabetes Prevention Program Outcomes Study. Lancet Diabetes Endocrinol. 2015;3(11):866-875.

17. Radovanovic CAT, Bevilaqua CA, Molena-Fernandes CA, Marcon SS. Intervenção multiprofissional em adultos com hipertensão arterial: ensaio clínico randomizado. Rev Bras Enferm. 2016;69(6):1005-1011.

18. Camolas J, Santos $O$, Mascarenhas M, Moreira P, Carmo I. Indivíduo: intervenção nutricional direcionada aos estilos de vida em indivíduos com obesidade. Acta Port Nutr. 2015;3(1):14-21.

19. Honsek C, Kabisch S, Kemper M, Gerbracht C, Arafat AM, Birkenfeld AL, et al. Fibre supplementation for the prevention of type 2 diabetesand improvement of glucose metabolism: the randomised controlledoptimal fibre trial (optifit). Diabetologia. 2018;61(6): 1295-1305.

20. Li Y, Hruby A, Bernstein AM, Ley SH, Wang DD, Chiuve SE, et al. Saturated fat as compared with unsaturated fats and sources of carbohydrates in relation to risk of coronary heart disease: a prospective cohort study. J Am Coll Cardiol. 2015;66(14):1538-1548.

21. Bressan J, Vidigal FC. Dieta na abordagem terapêutica da síndrome metabólica. R. Assoc Bras Nutr. 2014;6(1):55-60.

22. Bonito J. A dieta mediterrânica na prevenção de doenças da contemporaneidade: Uma revisão bibliográfica. Ver Bras Educ Saúde. 2016;6(1):27-35.

23. Saneei P, Fallahi E, Barak F, Ghasemifard N, Keshteli AH, Yazdannik AR et al. Adherence to the DASH diet and prevalence of the metabolic syndrome among Iranian women. Eur J Nutr. 2014;54(3):1-9. 